\title{
Lessons from History: Association of Porphyromonas Gingivalis (a Major Periodontopathic Bacteria) in Alzheimer's Disease Patients
}

\author{
Joao Carnio DDS, MS*, and Anna Tereza Carnio*1 \\ ${ }^{1}$ Private Practice, Londrina, Brazil, DDS Student at University of North Parana (Brasil) \\ Corresponding author: Joao Carnio, DDS, MS, Private Practice, Avenida Adhemar Pereira de Barros 131, \\ Jardim Bela, Suica 86050-190, Londrina, Parana, Brazil.
}

Citation: Joao Carni, MS, Lessons from History: Association of Porphyromonas Gingivalis (a Major Periodontopathic Bacteria) in Alzheimer's Disease Patients published in Med Science Journals.

Received Date: July 20, 2021 Accepted Date: Aug 06, 2021 Published Date: Aug 16, 2021

\begin{abstract}
Alzheimer's disease (AD), a fatal neurodegenerative condition that affects the elderly, is a serious health problem for geriatric subjects worldwide. AD incidence increases significantly with age. It is almost 50\% common in 85 -yearolds. [1] AD prevalence will rise as the population grows older and lives spans increase. It is estimated that around 14 million people will be affected by $A D$ in the next 50 years. Switching to newer treatments can help reduce the incidence of AD. These treatment options can be effective against potential risk factors and delay the onset.

What is the role of periodontitis in Alzheimer's disease?

This work aims to do a systematic, integrative review on published literature to evaluate if there is a link between Porphyromonas gumivalis (P. gingivalis), and Alzheimer's.

Part of (?) Part of (?)

P. gingivalis could serve as a therapeutic target for patients suffering from Alzheimer's disease. It also helps to reduce the severity and incidence of the condition. Patients with Alzheimer's disease could benefit from preventive dental care and the inhibition of neurotoxicity by $P$. gingivalis.
\end{abstract}

\section{Introduction}

Alzheimer's disease is the leading cause of dementia among seniors. Alzheimer's disease is a serious concern due to the increasing life expectancy. Alzheimer's disease is characterized by a brain disorder that can affect memory and cognitive function. This may be an early or sporadic form. It is common in $95 \%$ of Alzheimer's patients. Because peripheral inflammation can cause brain inflammation, it is widely believed that it contributes to the pathogenesis of Alzheimer's disease. The presence of pro-inflammatory cytokines like Interleukin 1-Beta, Tumor necrosis factor (TNF-), and Interleukin 6 is a sign that there is inflammation in the brains of patients with Alzheimer's disease. Amyloid-beta plaque $(\mathrm{Ab})$ and hyperphosphorylated Tau (2,7,8, respectively) are two hallmark proteins that can be seen in patients with Alzheimer's disease. Although the exact cause of Alzheimer's disease is unknown, other than the age-dependent risk, recent research has suggested that bacterial infection may be responsible. A growing number of studies have shown that periodontitis is linked to Alzheimer's disease.

One question remains: How can periodontitis be treated?

Are you a suspect in Alzheimer's disease Periodontitis is an oral multi-bacterial disease that causes chronic inflammation in the periodontal? Periodontal disease can cause systemic inflammation. It is thought to be caused by dysbiosis in oral bacteria. Porphyromonas gumivalis (P. gingivalis), plays a dominant role. This review will focus on P. gingivalis, a major periodontal disease, which has been suggested as a risk factor for the development of Alzheimer's. P gingivalis can be spread from the area of periodontal inflammation into systemic circulation. You can be inducible by simple tasks like brushing, flossing, and mastication as well as dental procedures such as Scaling or teeth extraction. P. gingivalis may cause systemic changes and be implicated in the onset or progression of various systemic diseases, including neurodegenerative, cardiovascular and rheumatoid. This review aims to identify a link between P. gingivalis (Alzheimer's disease) and this condition. It also explains how dentists can help patients with Alzheimer's by providing new information on oral care.

\section{Materials and Methods}

PICO model was used. Intervention (positive for periodontal bacteria), Patient (with Alzheimer's disease). P. gingivalis), Control, (patient with Alzheimer disease), and Outcome (association between P. gingivalis and patients with Alzheimer's). The question that is being asked is: Is P. gingivalis, which is a periodontal bacterium, linked to Alzheimer's disease? PRISMA protocol has inspired the use of flow diagrams in our methods. 
Mechanisms that promote inflammation to the brain. Two mechanisms in the brain that cause an increase in proinflammatory chemicals are systemic circulation (or neural pathways) and neural pathways (or both). Proinflammatory molecules can enter the brain via areas that lack blood brain barrier (BBB) in systemic circulation. These inflammatory molecules may also be able to enter brain areas with blood brain barrier (BBB) by:
(a) Fenestrated Capillaries of BBB
(b) Cytokine-specific transporters are used
(c) Increasing the permeability or BBB.
(d) The brain's endothelial cells are activated to make cytokine-inducing signals molecules like nitric oxide and prostanoids.

The brain reacts to proinflammatory molecules by increasing the amount of local proinflammatory chemicals or stimulating glial cells to produce additional proinflammatory substances. Neuronal pathway is another way that cytokines from peripheral sources could affect the brain. [20] Peripheral Cytokines can stimulate peripheral nerve afferent fibers, which results in an increase of brain cytokines. They also have the ability to use channels or compartments that are associated with peripheral nerves to enter brain. Another mechanism is the existence of CD14 receptors in the brain that can be activated by LPS derived invasive bacteria or AD AbP. This will in turn activate CD14 cells. These CD14 cells can be exposed to systemic circulation, such as the leptomeninges and circumventricular areas and the choroid plexus. This could increase brain cytokines, possibly contributing to the AD-related inflammatory burden.

\section{Methodology of research}

PUBMED was used to perform a bibliographic search. Different combinations of Keyswords were used: "Porphyromonas gumivalis" AND "Alzheimer's disease" OR "vascular dementia" ("periodontitis") OR "periodontitis".

Criteria for eligibility

Several kinds of publications were taken in consideration: Meta-analyses, experimental studies, study cases, clinical trials, in vitro and in vivo studies and integrative/systematic reviews. Only articles that dealt with P. gingivalis and Alzheimer's disease were chosen. They had to have been published between January 2008 and April 2020 in English or Portuguese. Because there were no researchers interested in this topic, the selection of articles was limited to the 20 most recent years. Only those articles were published in full and in digital form were elected. To assess the relevance of each, title, resume, and abstract were analyzed. These potentially eligible articles were then exanimated using a full-text analysis to determine if they were suitable for our study.

These were the exclusion and inclusion criteria

* Included were studies that explain the association between P. gingivalis and Alzheimer's disease.

* Included were studies describing the influence of P. gingivalis upon the onset Alzheimer's disease.

* Included were studies on mice and humans.

* Articles not in English or Portuguese before January 2008 were exclusions.

* Literature reviews were not included in the final results.

\section{Screening method}

After identifying eligible articles from various databases and platforms, we selected relevant articles to study the impact of P. gingivalis in Alzheimer's disease. Total number of articles found. All articles were compiled in Mendeley Citation Manager, where duplicates were removed. To select the correct publication that will fulfill the purpose of the study, a full evaluation of each publication was done. To remove irrelevant articles, the screening process included an analysis of title and abstract. All articles were then read in their entirety, to evaluate. Qualitative analysis is possible. To evaluate the articles, different factors were used: Authors, names, countries, years, country and type of study. Sample size, part tested, methodology used, conclusion, and result. The study's results showed that P. gingivalis was associated with Alzheimer's disease. Periodontitis and AD -- A correlation. This process is influenced by inflammation. Two possible mechanisms that could lead to AD progression are periodontitis and autoimmune disease.

There are two mechanisms that explain why AD and periodontitis can be associated.

The host response and periodontopathic microorganisms cause an increase of proinflammatory cytokines. This causes a wide range of pro-inflammatory and cytokines to be released into the systemic circulation, resulting in systemic/peripheral inflammation. These proinflammatory molecules can enter the cerebral regions and compromise the BBB. This can trigger activation or priming of microglial cells, which can lead to neuronal injury. This second mechanism is believed to be caused by the invasion of brain microorganisms in dental plaque biofilm. The microorganisms found in dental plaque can either enter the brain through peripheral nerves or bloodstream. These microorganisms, and their products, trigger an inflammation mechanism in the CNS. With substantial evidence, it is generally accepted that inflammation within the CNS can cause cognitive impairments such as AD. Cytokine-mediated interactions between neurons, glial cells and neurons are responsible for this inflammatory impairment. TNF-a and transforming growth factor $-b$ are some of the cytookines that are released by inflammation. Chemokines (monocyte chemotactic proteins, IL-8 and macrophage migration inhibitory factors, and monokine induced with g-interferon), have also been linked to serum and plasma biomarkers in AD pathogenesis. TNF-a, a key cytokine released during inflammation plays a significant role in neurodegenerative diseases. 
TNF-a increases the inflammation process, resulting in cell death, demyelination and BBB deterioration. TNF-a is a key player in neurodegenerative processes. The effects of cytokines as well as other proinflammatory molecules are markedly reduced by antiinflammatory drugs that are administered during inflammatory conditions. Research on mice models has shown that antiinflammatory agents have beneficial effects in the relief of neuroinflammation, and amyloid plaque formation. Nonsteroidal antiinflammatory agents also result in significant decreases in IL-1b levels and glial fibrillary acidsic protein in mice. Alzheimer's Disease Anti-inflammatory Prevention Trial (ADAPT), which examined the role of anti-inflammatory medications, hypothesized that their beneficial effects are only evident in the early stages of the disease. IL-1b levels were associated with cognitive decline in AD patients. Patients who had elevated levels of IL-1b before baseline showed greater cognitive decline rates over the 2-month period. This was compared to patients with lower levels. Dementia is also thought to be complex due to the interaction of genetics and systemic inflammation. High levels of blood inflammatory markers are associated with increased risk for dementia and higher incidences of cognitive impairment. Studies that were both longitudinal and cross-sectional have shown dementia in people with poor oral health. Periodontitis, which causes the body to produce inflammatory molecules in the systemic circulation, is thought to be a risk factor for developing various systemic diseases such as AD.

\section{Results:}

Between 2008 and 2020, the PubMed database contained 79 articles. Apply Study eligibility criteria: 32 articles were considered for consideration (studies on the relationship of P. gingivalis to periodontitis, major pathogen in periodontitis and Alzheimer' disease). 12 articles were selected for qualitative analysis after screening and applying inclusion and exclusion criteria. These studies were identified. The reviews not chosen were used to enrich and deepen our knowledge about the subject matter.

\section{Conclusions:}

Periodontitis and AD could be linked by inflammation. There are no studies in animals that specifically address the relationship between periodontal inflammation and AD. Periodontitis can infiltrate the systemic circulation and cause systemic disease. It is therefore advisable to prevent any further progression of periodontal disease.

\section{References}

1. Wyss-Coray T., Rogers J., Inflammation in Alzheimer disease-A brief review of the basic science and clinical literature. Cold Spring Harb. Perspect. Med. 2, a006346 (2012). [PMC free article] [PubMed] [Google Scholar]

2. Kaushal V., Dye R., Pakavathkumar P., Foveau B., Flores J., Hyman B., Ghetti B., Koller B. H., LeBlanc A. C., Neuronal NLRP1 inflammasome activation of Caspase-1 coordinately regulates inflammatory interleukin-1-beta production and axonal degenerationassociated Caspase-6 activation. Cell Death Differ. 22, 1676-1686 (2015). [PMC free article] [PubMed] [Google Scholar]

3. Mawanda F., Wallace R., Can infections cause Alzheimer's disease? Epidemiol. Rev. 35, 161-180 (2013). [PMC free article] [PubMed] [Google Scholar] 